\title{
A GENETICAL ANALYSIS OF DIVERSITY AND ASYMMETRY IN FINGER RIDGE COUNTS
}

\author{
N. G. MARTIN, ${ }^{*} \dagger$ J. L. JINKS, $\dagger$ H. S. BERRY, $\dagger$ and D. Z. LOESCH $\ddagger$ \\ * Department of Population Biology, Research School of Biological Sciences, Australian \\ National University, Canberra, Australia; † Department of Genetics, University of \\ Birmingham, Birmingham, England.
}

Received 8.i.82

\section{SUMMARY}

The genetics of asymmetry and diversity of finger ridge counts in man has been examined by jointly regressing the individual counts of each hand on to the mean values summed over left and right hands in a sample of twins. The resulting asymmetry terms are largely under environmental control but with a small significant genetic component. The diversity items show a larger degree of genetic control with a suggestion of dominance or additive $\times$ additive epistasis.

\section{INTRODUCTION}

IT is well known that there is considerable diversity between the mean ridge counts of individual fingers in man, with digits I and IV having the highest counts and digits II and III the lowest (e.g., Holt, 1968; Sievogel et al., 1979). Part of this diversity is confounded with differences in the distribution of pattern types from finger to finger. There are also asymmetric differences between corresponding fingers on the two hands, particularly the thumb where the right hand count is usually greater than the left.

A number of authors have explored the genetics of individual differences in ridge count diversity and asymmetry. Most have employed the socalled diversity index $S / \sqrt{10}$ first used by Holt (1958), where $S^{2}=$ $\sum_{i=1}^{10} q_{i}^{2}-\left(\sum q_{i}\right)^{2} / 10$ and $q_{1} \cdots q_{10}$. are the separate digital ridge counts. However, this index confounds differences due to diversity between the five means of pairs of corresponding digits and to asymmetry within these pairs of digits. In a family study of $S / \sqrt{10}$, Holt (1960) found correlation coefficients of 0.73 for $\mathrm{MZ}$ twins and 0.22 for $\mathrm{DZ}$ twins, sibs and also for parents and offspring. It is clear that there is a substantial genetic component in the inheritance of this index but although the collateral data suggest a large non-additive genetic component, the parent-offspring correlation should be smaller than the sibling correlation to be consistent with an hypothesis of dominance. Epistatic interactions between additive gene effects at different loci, however, would produce this pattern of correlations (Loesch et al., 1982).

The compound nature of $S / \sqrt{10}$ was recognised by Jantz $(1974,1976)$ who defined separate diversity and asymmetry indices. He showed considerable racial variation in these indices, with hybrid values falling between those of the parental races, and concluded that both diversity and asymmetry were under partial genetical control. Poland.

$¥$ Permanent address: Department of Genetics, Psychoneurological Institute, Warsaw, 
Holt (1954) examined the genetics of the difference between right and left hand total ridge counts. She found all familial correlations close to zero and concluded that nearly all variation in asymmetry was environmentally determined. In contrast, Singh (1970), using a slightly different index of asymmetry, found heritabilities in the range $0 \cdot 20-0 \cdot 44$.

In the present study, we derive a number of separate indices of diversity and asymmetry from a joint regression analysis of the kind first introduced by Yates and Cochran (1938) and developed by Perkins and Jinks (1968) for the analysis of genotype $\times$ environment interaction. We then use the classical twin design to examine their sources of variation.

\section{MAterials AND METHOdS}

Finger ridge counts for 221 pairs of Polish monozygotic and like-sex dizygotic twins were used in the analysis. The data and the sample are described in detail elsewhere (Martin et al., 1982a).

\section{(i) Joint regression analysis}

The diversity index $S / \sqrt{10}$, used by Holt (1968) and others, measures deviations from the mean of an individual's ten counts and neglects the fact that there are characteristic population mean values for each finger. A more interesting question is the extent to which an individual's counts deviate from the characteristic ranking of finger population means. Deviations from this ranking may be congruent in corresponding fingers or asymmetric.

For each individual we can carry out a joint regression analysis, the five counts of each hand being the two sets of dependent measurements $\left(y_{i L}\right.$ and $\left.y_{i R}\right)$ and the population means for each finger, (summed across both hands) the set of five independent values $\left(x_{i}\right)$. Different $x_{i}$ 's are used for males and females.

Of course, the five $x_{i}$ values are not strictly independent since, instead of population means, we use the total sample means for males and females. These are given in table 1, separately for left and right hands and summed over the two hands in both males and females. There are slight differences in the ranking of fingers between hands but the ascending ranking of fingers, summed over counts for both hands is 23541 for males and 25341 for females. For each individual we may now regress the two sets of five counts on the male or female set of sample means:

\begin{tabular}{|c|c|c|c|c|c|}
\hline & \multirow{3}{*}{$\begin{array}{l}\text { Sample means } \\
\qquad x_{i L}+x_{i R}\end{array}$} & \multicolumn{4}{|c|}{ Finger counts of $i$ th individual } \\
\hline & & \multirow{2}{*}{$\begin{array}{c}\text { Left } \\
y_{i L}\end{array}$} & \multicolumn{2}{|c|}{ Right Sum } & \multirow{3}{*}{$\left(=y_{i L}+y_{i R}\right)$} \\
\hline & & & $y_{i R}$ & $y_{i T}$ & \\
\hline Fing & & & & & \\
\hline 1 & $x_{1}$ & $y_{1 L}$ & $y_{1 R}$ & $y_{1 T}$ & \\
\hline 2 & $\cdot$ & $\cdot$ & $\cdot$ & $\cdot$ & \\
\hline 3 & $\cdot$ & $\cdot$ & · & · & \\
\hline 4 & . & . & - & . & \\
\hline 5 & $x_{5}$ & $y_{5 L}$ & $y_{5 R}$ & $y_{5 T}$ & \\
\hline
\end{tabular}


TABLE 1

Mean finger ridge counts and s.e.'s for the twin sample

\begin{tabular}{llll}
\multicolumn{3}{c}{ Males $n=244$} \\
L1 & $17 \cdot 00 \pm 0.39$ & R1 & $19.54 \pm 0.35$ \\
L2 & $12.33 \pm 0.39$ & R2 & $13.05 \pm 0.45$ \\
L3 & $13.84 \pm 0.36$ & R3 & $12.74 \pm 0.38$ \\
L4 & $17.76 \pm 0.33$ & R4 & $17.66 \pm 0.34$ \\
L5 & $14.30 \pm 0.29$ & R5 & $14.32 \pm 0.30$
\end{tabular}

Left total $75 \cdot 23 \pm 1 \cdot 41$

Right total $77 \cdot 31 \pm 1.45$

$\begin{array}{ll}\text { L1 } & 15 \cdot 32 \pm 0.42 \\ \text { L2 } & 11 \cdot 48 \pm 0.47 \\ \text { L3 } & 12 \cdot 71 \pm 0.44 \\ \text { L4 } & 16 \cdot 10 \pm 0.45 \\ \text { L5 } & 12 \cdot 22 \pm 0.36\end{array}$

Females $n=198$

Left total $67 \cdot 82 \pm 1.78$

\section{R1}

R2

R3

R4

R5

$18 \cdot 07 \pm 0 \cdot 39$

$11.99 \pm 0.53$

$12.63 \pm 0.41$

$16 \cdot 27 \pm 0 \cdot 41$

$12 \cdot 34 \pm 0 \cdot 40$

Right total $71 \cdot 29 \pm 1 \cdot 75$

\begin{tabular}{lccc} 
& \multicolumn{2}{c}{ Left and Right } & \\
& Males & & Females \\
1 & $18 \cdot 27$ & 1 & 16.69 \\
2 & 12.69 & 2 & 11.73 \\
3 & 13.29 & 3 & 12.67 \\
4 & 17.71 & 4 & 16.18 \\
5 & 14.31 & 5 & 12.28
\end{tabular}

This joint regression analysis yields the following terms.
Item
df
Analysis of Variance
(1) $L \vee R$
$1 \frac{1}{10}\left(\sum y_{R}-\sum y_{L}\right)^{2}$
(2) Joint Regression
$1 \frac{\left(\mathrm{SP} x y_{T}\right)^{2}}{2 \mathrm{SS} x}$
(3) Heterogeneity
$1 \quad \frac{\left(\mathrm{SP} x y_{L}\right)^{2}}{\mathrm{SS} x}+\frac{\left(\mathrm{SP} x y_{R}\right)^{2}}{\mathrm{SS} x}-(2)$
(4) Joint remainder
$3 \quad \mathrm{SS}_{T}-(2)$
(5) Heterogeneity
$3 \quad\left(\mathrm{SS} y_{L}-\operatorname{Reg} \mathrm{SS}_{L}\right)+\left(\mathbf{S S} y_{R}-\operatorname{Reg} \mathrm{SS}_{R}\right)-(4)$ of remainder

Where,

$$
\text { Regression } \mathrm{SS}_{L}\left(\operatorname{Reg} \mathrm{SS}_{L}\right)=\frac{\left(\mathrm{SP} x y_{L}\right)^{2}}{\mathrm{SS} x} \quad\left(\text { similarly for } \operatorname{Reg} \mathrm{SS}_{R}\right)
$$

and

$$
\operatorname{SS} y_{T}=\frac{1}{2}\left(\sum y_{T}^{2}-\frac{\left(\sum y_{T}\right)^{2}}{5}\right)
$$

(SS denotes sum of squares, SP denotes sum of products). 
The five terms in the analysis of variance can be interpreted as follows:

(a) $L \vee R$. This estimates the difference between left hand and right hand total ridge counts. It is similar to the character in Holt's (1968) analysis of asymmetry.

(b) Joint regression. This is the amount of variation in the ridge counts of an individual which is accounted for by the finger ridge counts on both hands having the same regression on the population means. i.e., the extent to which an individual has the same order of counts as the average, this pattern being the same in both hands. If this were the only significant item in the analysis, a schematic plot of an individual's counts against the population means might resemble fig. 1(a).

(c) Heterogeneity of regression. The extent to which the separate regression slopes for the left and right hands differ from the joint regression slope is measured by the heterogeneity of regression. If this item and the joint regression item only were significant a schematic plot might resemble fig. 1(b).

(d) Joint remainder. If the counts of an individual differ from the joint regression line, but with corresponding fingers on left and right hands having the same deviations from average, then there will be a joint remainder term. This is illustrated in fig. 1(c) where the solid lines denote the joint regression.

(e) Heterogeneity of remainder. Where differences in finger ridge counts cannot be accounted for by regression, and the deviations from the regression line are different in left and right hands, such differences will contribute to the heterogeneity of remainder. This is illustrated in fig. 1(d).

Note that in fig. 1(c) and 1 (d) we have assumed that there is no heterogeneity of regression, in fig. 1(c) there would be no heterogeneity of remainder and in fig. 1(d) there would be no joint remainder.

Of the five items we may regard the joint regression and joint remainder items as measures of diversity, while the $L \vee R$ and the two heterogeneity items are measures of asymmetry.

This analysis has been carried out for each of the 442 individuals. While it is valid to test the significance of all mean squares except the heterogeneity of remainder, the degrees of freedom are too small to make this a useful exercise. It is not valid to sum the mean squares from the individual regression analyses in order to test their significance in the population but nevertheless, the average mean squares are shown in table 2.

\section{(ii) Genetical analysis of variation}

Since none of the terms is negligible, we would like to dissect the causes of each of the five types of variation in finger ridge counts. We know from

TABLE 2

Average values of mean squares from individual regression analyses

$\begin{array}{lcc} & \text { Males } & \text { Females } \\ & n=244 & n=198 \\ R \vee L \text { bias } & 11.88 & 11 \cdot 14 \\ \text { Joint Regression } & 75.03 & 66.76 \\ \text { Heterogeneity of Regression } & 10 \cdot 14 & 10.24 \\ \text { Joint Remainder } & 22.40 & 19.89 \\ \text { Heterogeneity of Remainder } & 7.98 & 9.38\end{array}$



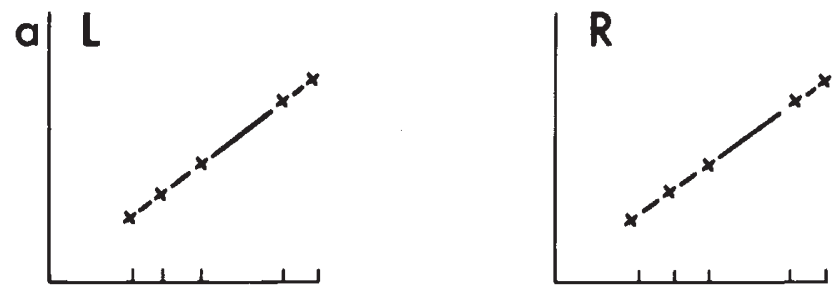

Joint regression

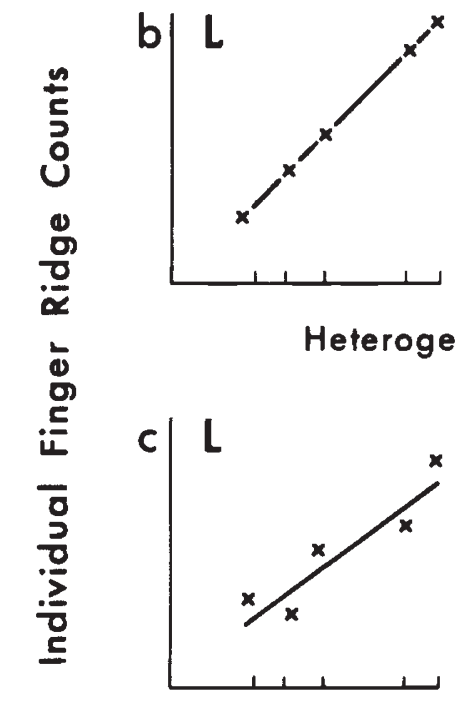

$\mathbf{R}$

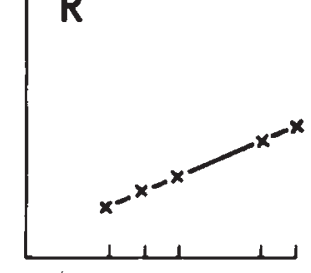

eity of regression 

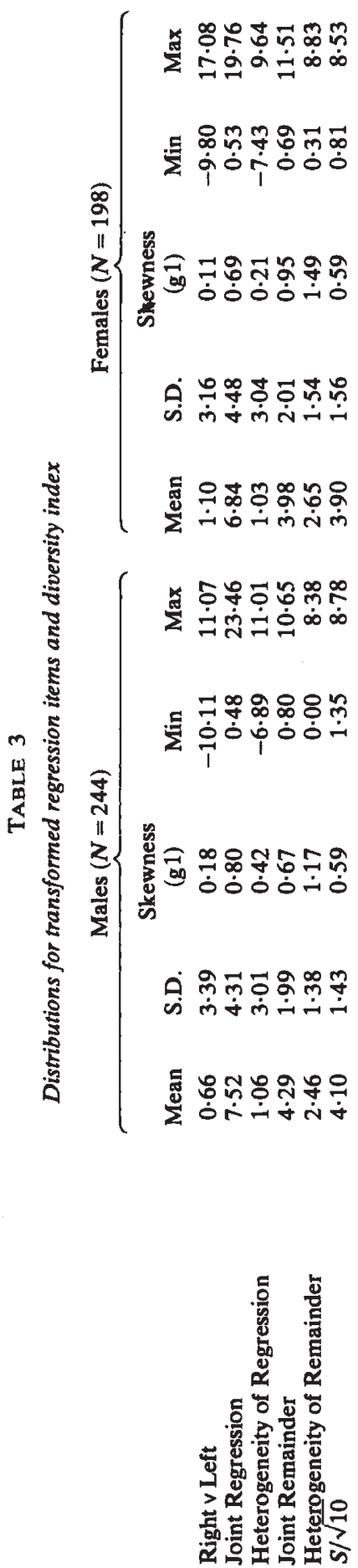
Distributions of the individual mean squares are highly skewed and more reasonable distributions can be obtained by taking the square roots of the mean squares. Since we are interested not only in the magnitude of right-left differences but also in their direction, the $\sqrt{R \vee L}$ term was given the sign of $(R-L)$ and the heterogeneity of regression term was given the sign of $\left(b_{R}-b_{L}\right)$ where $b_{R}$ and $b_{L}$ are the regression coefficients for right and left hands respectively. The distributions of these five transformed variables are given in table 3, separately for males and females. Also given, for comparison, is the distribution of $S / \sqrt{10}$.

We investigate the causes of variation by fitting models to mean squares using the method of weighted least squares. The method and the models employed are described elsewhere (Martin et al., 1981a). The model for twin mean squares, with parameters for individual environmental variation $\left(E_{w}\right)$, additive genetic $\left(D_{R}\right)$ and dominance $\left(H_{R}\right)$ variation is shown in table 4.

TABLE 4

Model for mean squares of twins reared together

$\begin{array}{cccc} & E_{w} & D_{R} & H_{R} / I_{R} \\ \text { MZ between } & 1 & 1 & \frac{1}{2} \\ \text { within } & 1 & 0 & 0 \\ \text { DZ between } & 1 & \frac{3}{4} & \frac{5}{16} \\ \text { within } & 1 & \frac{1}{4} & \frac{3}{16}\end{array}$

Mean squares for the five transformed regression terms and for $S / \sqrt{10}$ are given in table 5. Three models of increasing complexity have been fitted to the data. The first tests the hypothesis that all variation is accounted for by individual environmental differences $\left(E_{w}\right)$, i.e., there is no variation attributable to differences between families (mean square $b$ in table 5) from any cause. Where this first model fails, as it will do if there are differences between families, the second model $\left(E_{w}\right.$ and $\left.D_{R}\right)$ tests the hypothesis that all of the heritable variation is additive $\left(D_{R}\right)$. Where this model in turn fails the third model $\left(E_{w}, D_{R}\right.$ and $\left.H_{R}\right)$ which extends the heritable variation to include non-additivity $\left(H_{R}\right)$ is fitted. Because the expected contributions for dominance $\left(H_{R}\right)$ and additive $\times$ additive epistatic interactions $\left(I_{R}\right)$ are completely confounded if only $\mathrm{MZ}$ and $\mathrm{DZ}$ twins are available (Mather, 1974) our designation of the non-additive genetic variance here as $H_{R}$ is quite arbitrary. The results of fitting these three models separately to the male and female data and jointly to all eight statistics are shown in table 6. For a given model, the heterogeneity of the fit over sexes can be calculated by subtracting the sum of the male and female chi-square values from the joint chi-square and similarly for their degrees of freedom.

\section{RESULTS OF MODEL FITTING}

(i) $R \vee L$

Variation in total $R \vee L$ asymmetry appears to be entirely environmentally controlled in females but under partial genetical control in males. The heterogeneity between sexes for the $E_{w} D_{R}$ model is $\chi_{2}^{2}=7 \cdot 54$. Herita- 
400 N. G. MARTIN, J. L. JINKS, H. S. BERRY AND D. Z. LOESCH

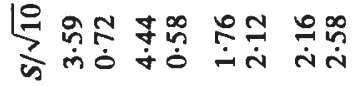

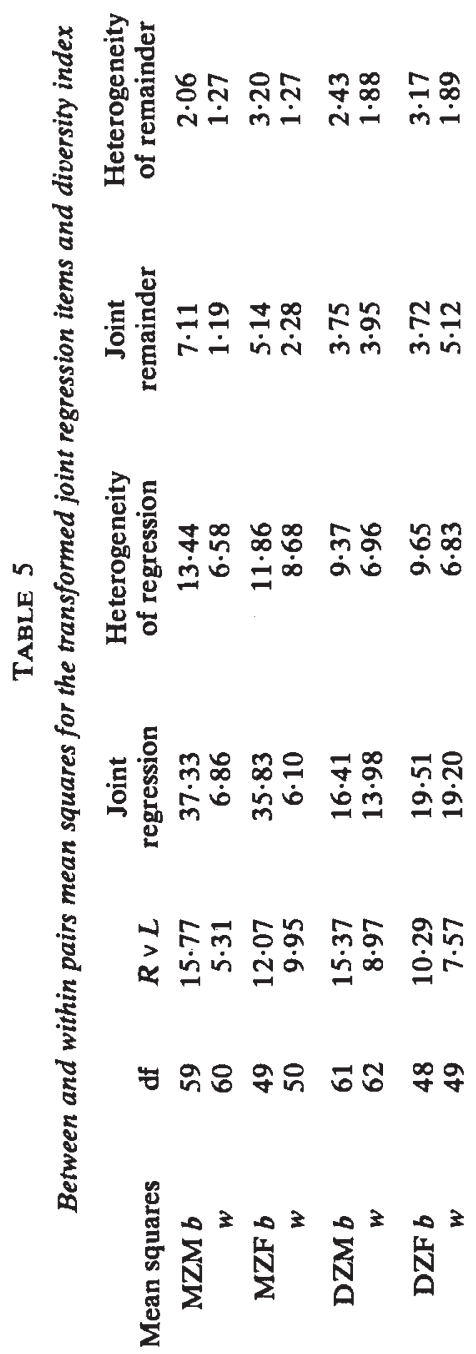


bility, calculated as $\frac{1}{2} D_{R} /\left(E_{w}+\frac{1}{2} D_{R}\right)$ is $0.52 \pm 0.08$ for males but is not significantly different from zero in females.

\section{(ii) Joint regression}

The fit of the $E_{w} D_{R}$ model is homogeneous over sexes but it is barely consistent with the data. There is a moderately high heritability of 0.64 for this model, but the model including dominance appears more appropriate. The negative and non significant estimates of $D_{R}$ and the large, positive and significant estimates of $H_{R}$ reflect the high negative correlation between $D_{R}$ and $H_{R}$ when estimated from MZT's and DZT's. Since an environmental difference between families $\left(E_{b}\right)$ would have inflated $D_{R}$ by $6 E_{b}$ and defiated $H_{R}$ by $8 E_{b}$ it is clear that $E_{b}$, which is not included in the model, must be very small or zero.

\section{(iii) Heterogeneity of regression}

As with the $R \vee L$ asymmetry item, the female data are consistent with a purely environmental hypothesis while the male data indicate some degree of additive genetical control with a heritability of 0.32 for this source of asymmetry. There is however no difference between sexes, and combined they give a low heritability of $0 \cdot 25$.

\section{(iv) Joint remainder}

The causes of variation are similar to those for joint regression. There is a difference between sexes with a moderately high degree of genetic control in males with a heritability of 0.66 and a much lower one in females $(0 \cdot 26)$. There is also strong evidence that dominance/epistasis is important. Again, however, $D_{R}$ and $H_{R}$ reflect the high negative correlation between them and the negative $D_{R}$ and large positive $H_{R}$ leave little doubt that $E_{b}$ is very small or zero.

\section{(v) Heterogeneity of remainder}

The male data are consistent with solely environmental variation but both male and female data are homogeneous in the adequacy of the $E_{w} D_{R}$ model and a heritability of $0 \cdot 37$.

$$
\text { (vi) } S / \sqrt{10}
$$

This has a pattern of variation very similar to that for the joint regression and joint remainder items. The $E_{w} D_{R}$ model is actually inadequate as an explanation of variation in both sexes. The addition of dominance/epistasis improves the fit of the model quite dramatically leading to the now familiar pattern of a negative $D_{R}$ and a large positive $H_{R}$ reflecting once more a negative correlation between the estimates and the absence of an $E_{b}$ component.

\section{Discussion}

Despite a number of inconsistencies in the results of model fitting, particularly between males and females, several points emerge clearly. The 
402 N. G. MARTIN, J. L. JINKS, H. S. BERRY AND D. Z. LOESCH

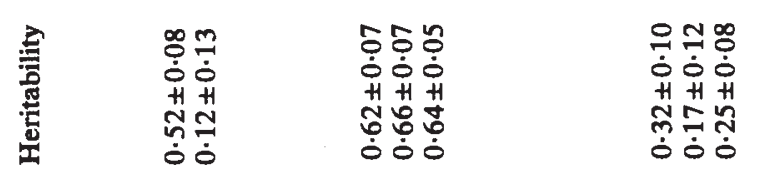

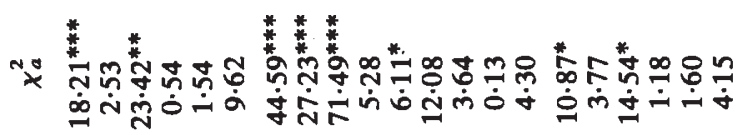

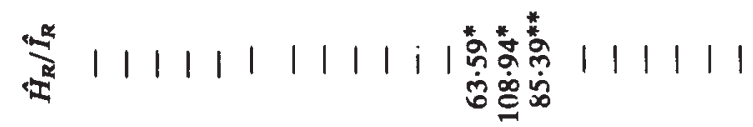

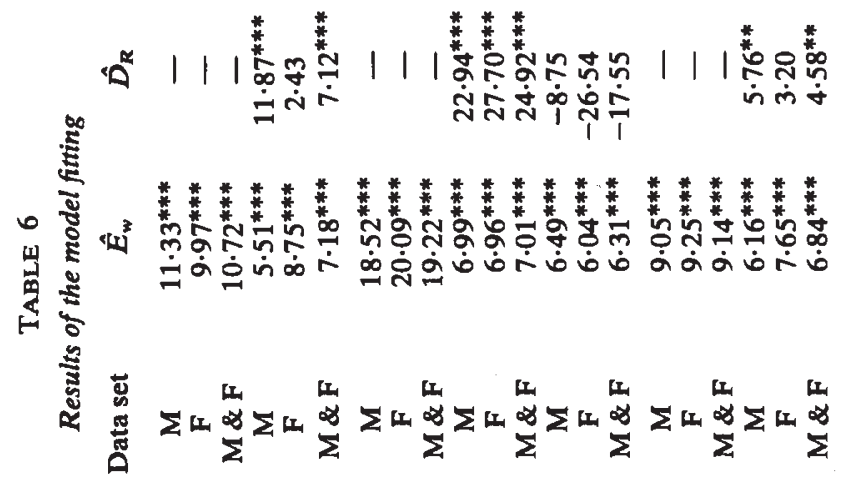

$\frac{\bar{\sigma}}{\frac{0}{2}}$

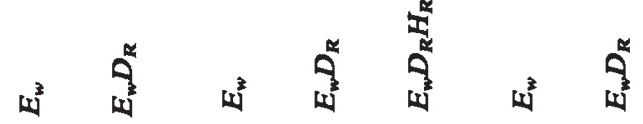

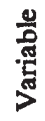

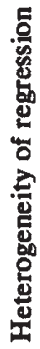




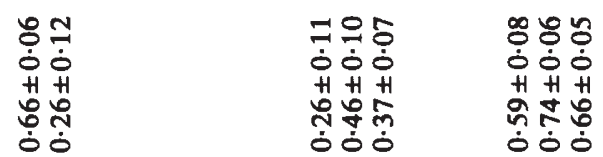

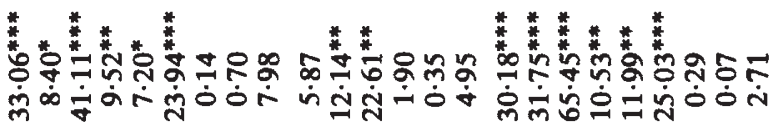

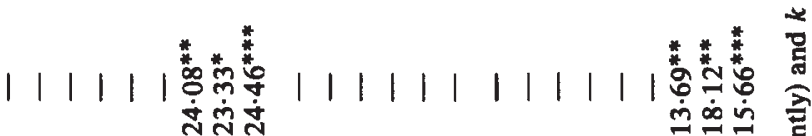

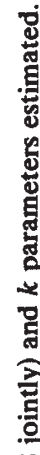

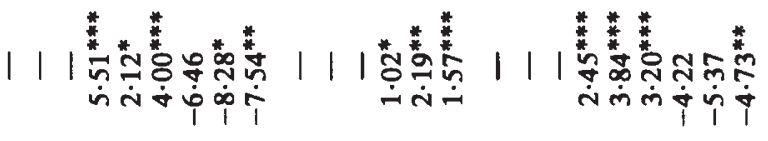

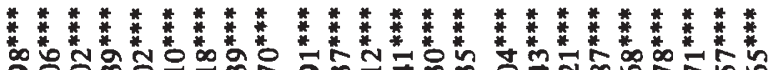

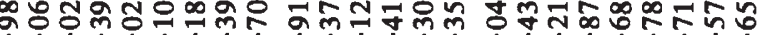
i் $\dot{\sim} \dot{\sim} \dot{m} \dot{\sim} \dot{\sim} \dot{\sim} \dot{-} \dot{\sim} \dot{\sim} \dot{-} \dot{\sim} \dot{\sim} \dot{N} \dot{0} \dot{0} \dot{0} \dot{0} \dot{0}$

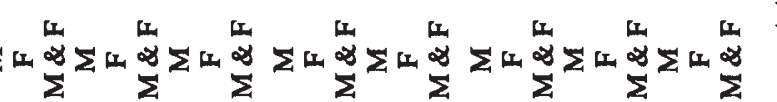

$$
\text { 造 }
$$

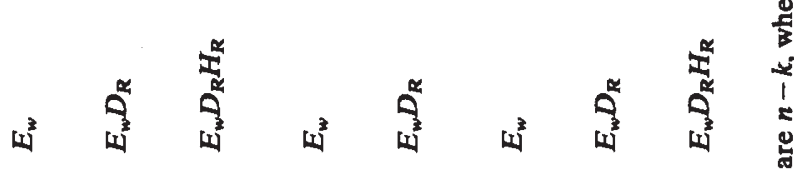

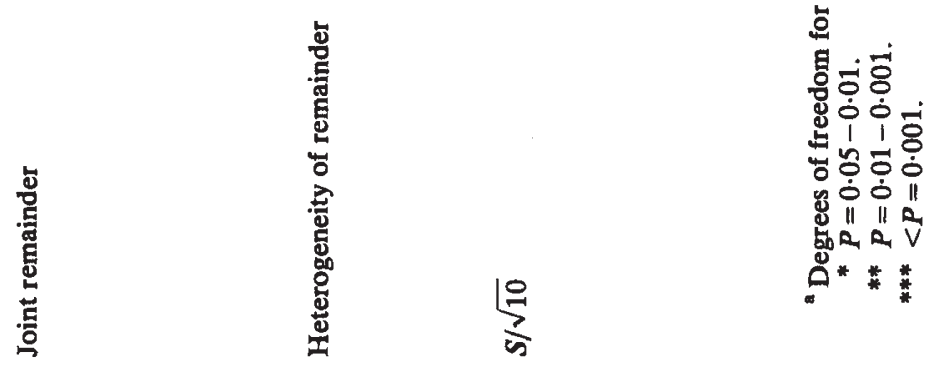


three asymmetry items, $R \vee L$, heterogeneity of regression and heterogeneity of remainder appear to be largely under environmental control. However, there does appear to be some genetical variation, more so in males than in females, for each of these characters. Our results agree with those found by Singh (1970) for his asymmetry index but contrast with Holt's finding that the total $R-L$ value shows no genetical variation at all. There is no evidence that genetical non-additivity plays a part in any of our measures of left-right differences. Loesch and Swiatkowska (1978) also found evidence for some genetic contribution to asymmetry in the distribution of patterns on fingers.

Variation in $R \vee L$ asymmetry is obviously a composite of variation in asymmetry for each of the five fingers. It is possible that this variation may be greater in some fingers than in others. It is also possible that the causes of variation in asymmetry may differ from finger to finger, being largely genetic for some and largely environmental for others. This possibility is explored elsewhere (Loesch and Martin, 1982). However, it should be noted that whatever the differences in size or causes of variation in asymmetry, these bear no necessary relationship to the mean value of asymmetry for a finger. Thus the fact that Jantz (1979) and others have termed the asymmetry in the thumb counts "directional" (i.e., $(\overline{R-L})>0$ ) and in other fingers "fluctuating" (i.e., $(\overline{R-L}=0)$ says nothing whatever about the causes of variation in asymmetry for that finger.

On the other hand the measures of diversity, joint regression and joint remainder from the regression analysis, and the diversity index, $S / \sqrt{10}$, advocated by Holt, all show a greater degree of genetical determination. However, in these variables the mean squares are anomalous in that (except for Joint Regression in $\mathrm{DZ}$ males) the $\mathrm{DZ}$ within pairs mean squares are all greater than their corresponding between pairs mean squares. We have already noted that Holt's DZ and sib correlations are very low in comparison with the $\mathrm{MZ}$ correlations but in our case most of the $r_{\mathrm{Dz}}$ 's would actually be negative although not significantly different from zero. This is inconsistent with a between family environmental component $\left(E_{b}\right)$ and it has no ready explanation in terms of the genetical models considered here but may be indicative of a high degree of genetical non-additivity such as dominance, or more probably, additive $\times$ additive epistasis (Loesch et al., 1982). If this is the case, then the results of this analysis are in agreement with the univariate and multivariate analyses reported in our earlier papers (Martin et al., 1981 $a, b$ ). Dominance has also been invoked to explain diversity of pattern type by de Wilde and Amesz-Voorhoeve (1979).

The genetical analysis of covariation between finger ridge counts (Martin et al., 1982b) provided evidence for a number of independent genetical factors determining the pattern of finger ridge counts. The action of these genetic factors was not perfectly consistent on both hands. In the present paper the same data has been analysed again by partitioning the available degrees of freedom in quite different ways. It has perhaps shown even more directly that variation in the ranking of finger ridge counts across both hands is largely under genetical control. There is also a genetic component in asymmetic variations between hands but environmental factors are more important. 


\section{REFERENCES}

DE WILDE, A. G., AND AMESZ-VOORHOEVE, W. H. M. 1979. Fingerprints: classifications, correlations and inheritance. In Wertelecki, W., and Plato, Ch. (eds.) Dermatoglyphics Fifty Years Later, The National Foundation - March of Dimes, Alan R. Liss, Inc., New York, pp. 95-118.

HOLT, S. B. 1954. Genetics of dermal ridges: bilateral asymmetry in finger ridge counts. Ann. Eugen. London, 18, 211.

HOLT, S. B. 1958. Genetics of dermal ridges: The relation between total ridge count and the variability of counts from finger to finger, Ann. Hum. Genet., London, 22, 323.

HOLT, S. B. 1960 . Genetics of dermal ridges: familial correlations for $(S / \sqrt{10})$, a measurement of the diversity of ridge counts from finger to finger. Ann. Hum. Genet., London, 24, 253.

HOLT, S. B. 1968. The Genetics of Dermal Ridges. Charles C. Thomas, Springfield, Illinois. JANTZ, R. L. 1974. Finger ridge counts and inter-finger variability in Negroes and Whites. Hum. Biol., 46, 663 .

JANTZ, R. L. 1976. The relation between total finger ridge count and variability of counts from finger to finger. Genetic implications of racial variation. Ann. Hum. Genet., London, $40,221$.

JANTZ, R. L. 1979. On the levels of dermatoglyphic variation. In Wertelecki, W., and Plato, Ch. (eds.) Dermatoglyphics - Fifty Years Later, The National Foundation - March of Dimes, Alan R. Liss, Inc., New York, pp. 53-61.

LOESCH, D., AND MARTIN, N. G. 1982. Directional and absolute asymmetry of digital ridge counts (submitted for publication.)

LOESCH, D., MARTIN, N. G., HEATH, A. C., AND EAVES, L. J. 1982. Evidence for polygenic epistatic interactions in man (submitted for publication.)

LOESCH, D., AND SWIATKOWSKA, Z. 1978. Dermatoglyphic total patterns on palms, fingertips and soles in twins. Ann. Hum. Biol., 5, 409.

MARTIN, N. G., LOESCH, D. Z., JARDINE, R., AND BERRY, H. S. 1982a. Evidence for directional dominance in the genetics of finger ridge counts. Annals of Human Biology (In Press).

MARTIN, N. G., EAVES, L. J., AND lOESCH, D. Z. 1982b. A genetical analysis of covariation between finger ridge counts. (Submitted for publication.)

MATHER, K. 1974. Non-allelic interactions in continuous variation of randomly breeding populations. Heredity, 32, 414.

PERKINS, J. M., AND JINKS, J. L. 1968. Environmental and genotype $\times$ environmental components of variability. III. Multiple lines and crosses. Heredity, 23, 339.

SIERVOGEL, R. M., ROCHE, A. F., AND ROCHE, E. M. 1979. The identification of developmental fields using digital distributions of fingerprint patterns and ridge counts. In Wertelecki, W., and Plato, Ch. (eds.) Dermatoglyphics - Fifty Years Later, The National Foundation - March of Dimes, Alan R. Liss, Inc., New York, pp. 135-147.

SINGH, s. 1970. Inheritance of asymmetry of finger ridge counts. Hum. Hered., 20, 403.

YATES, F., AND COCHRAN, W. G. 1938. The analysis of groups of experiments. J. Agric. Sci., 28, 556. 